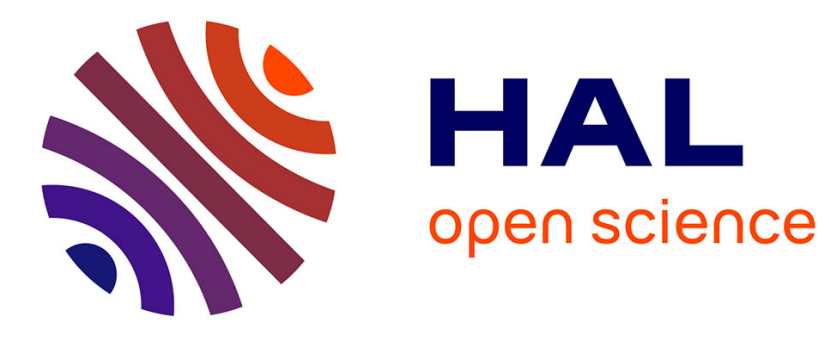

\title{
On the smooth values of shifted almost-primes
}

Xiaodong Lü, Zhiwei Wang, Bin Chen

\section{To cite this version:}

Xiaodong Lü, Zhiwei Wang, Bin Chen. On the smooth values of shifted almost-primes. International Journal of Number Theory, 2019, 15 (01), pp.1-9. 10.1142/S1793042118501683 . hal-03283539

\section{HAL Id: hal-03283539 \\ https://hal.science/hal-03283539}

Submitted on 11 Jul 2021

HAL is a multi-disciplinary open access archive for the deposit and dissemination of scientific research documents, whether they are published or not. The documents may come from teaching and research institutions in France or abroad, or from public or private research centers.
L'archive ouverte pluridisciplinaire HAL, est destinée au dépôt et à la diffusion de documents scientifiques de niveau recherche, publiés ou non, émanant des établissements d'enseignement et de recherche français ou étrangers, des laboratoires publics ou privés. 


\title{
ON THE SMOOTH VALUES OF SHIFTED ALMOST-PRIMES
}

\author{
BIN CHEN, XIAODONG LÜ AND ZHIWEI WANG
}

\begin{abstract}
Denote by $P^{+}(n)$ (resp. $\left.P^{-}(n)\right)$ the largest (resp. the smallest) prime factor of the integer $n \geqslant 1$. In this paper, we prove a lower bound of almost-primes $n \leqslant x$ with $P^{-}(n)>x^{1 / v}, v>4$ such that $P^{+}(n-a) \leqslant x^{1 / u}$ for $1 \leqslant u \ll 1, a \in \mathbb{Z}, a \neq 0$. As an application, we study two patterns on the largest prime factors of consecutive integers with one of which without small prime factor. This is a corrigendum to the original paper [18].
\end{abstract}

\section{INTRODUCTION}

For each integer $n \geqslant 1$, let $P^{+}(n)$ denote the largest prime factor of $n$ with the convention that $P^{+}(1)=1$. The distribution of the largest prime factors of shifted primes $P^{+}(p-a)$ with $a \in \mathbb{Z}, a \neq 0$ have been studied by many mathematicians, such as Erdôs [5], Goldfeld [12], Pomerance [20], Fouvry [9], Baker and Harman [1], Banks and Shparlinski [2]. Define

$$
\pi(x, y):=\sum_{\substack{p \leqslant x \\ P^{+}(p-a) \leqslant y}} 1 .
$$

In 2008, Granville [13] stated that the asymptotic formula

$$
\pi(x, y) \sim \rho(u) \pi(x), \quad y=x^{1 / u}
$$

follows for all fixed $u \geqslant 1$ from a weak form of the Elliott-Halberstam conjecture, a wellbelieved conjecture in analytic number theory. Here $\rho(u)$ is the Dickman-de Bruijn function, and $\pi(x)$ denotes the number of primes not exceeding $x$. Recently the second named author [25] provided the details for proof of (1.2) under the Elliott-Halberstam conjecture.

In this paper, we consider the analogue problem via replacing prime $p$ by almost-prime (with a limited number of prime factors). For $a \in \mathbb{Z}, y \leqslant x$ and $z \leqslant x$, we define

$$
\Phi(x, y, z):=\sum_{\substack{n \leqslant x, P^{-}(n)>z \\ P^{+}(n-a) \leqslant y}} 1,
$$

where $P^{-}(n)$ denotes the smallest prime factor of integer $n$ with the convention $P^{-}(1)=\infty$. For the case $a=0$, we may refer to the work of Friedlander [11] and Saias [21, 22, 23]. Here we only consider the case $a \neq 0$. Considering the following formula for almost-primes (see [24, Chapitre III.6]):

$$
\Phi(x, z):=\sum_{\substack{n \leqslant x \\ P^{-}(n)>z}} 1=\frac{x \omega(v)-z}{\log z}+O\left(\frac{x}{(\log z)^{2}}\right), \quad z=x^{1 / v}
$$

for $x \geqslant z \geqslant 2$, where $\omega(v)$ is the Buchstab function, we may guess that

$$
\Phi(x, y, z) \sim \rho(u) \omega(v) \frac{x}{\log z}, \quad y=x^{1 / u}, z=x^{1 / v}
$$

Date: July 11, 2021.

2000 Mathematics Subject Classification. 11N05, 11N36.

Key words and phrases. shifted almost-primes, friable numbers, sieve methods. 
for some $u, v \geqslant 1$.

It's not difficult to prove (1.4) with $1 \leqslant v, 1 \leqslant u \ll 1$ under the Elliott-Halberstam conjecture for almost-primes (replacing primes by almost-primes) by following the argument of Lemma 4.1 in [25]. We leave the details to the interested reader.

In this paper, we first prove an unconditional result on the estimate of $\Phi(x, y, z)$ for some $u, v \geqslant 1$.

Theorem 1. For $x \rightarrow \infty$ and $y=x^{1 / u}, z=x^{1 / v}$, there exists a strictly positive constant $c_{1}(u, v)$ such that

$$
\Phi(x, y, z) \geqslant c_{1}(u, v) \frac{x}{\log x}
$$

for $v>4,1 \leqslant u \ll 1$.

Remark. With the same method to (1.5), we can obtain an upper bound

$$
\Phi(x, y, z) \leqslant c_{2}(u, v) \frac{x}{\log x}
$$

for $1 \leqslant v \ll 1,1 \leqslant u \ll 1$, where $c_{2}(u, v)$ is a strictly positive constant. In fact, (1.6) is the case " $q=1$ " of Theorem 1 in [17]. An application of the upper bound (1.6) is to study the density of shifted primes with large prime factors. For $0<\theta<1$, we define

$$
T_{\theta}(x):=\left|\left\{p \leqslant x: P^{+}(p-1) \geqslant p^{\theta}\right\}\right|,
$$

and we want to estimate $T_{\theta}(x)$ with a good lower bound (see [19], [3] and [6]). As in the very recent work of Liu, Wu and Xi [17], with the help of (1.6), for $x \rightarrow \infty$ we have the following lower bound

$$
T_{\theta}(x) \geqslant \begin{cases}(1-4 \rho(1 / \theta)+o(1)) \frac{x}{\log x} & \text { for } 0<\theta \leqslant \theta_{0}, \\ (1-\theta+o(1)) \frac{x}{\log x} & \text { for } \theta_{0}<\theta \leqslant 1 / 2,\end{cases}
$$

where $\theta_{0} \approx 0.3734$.

As an application of Theorem 1, we treat a problem on the largest prime factors of consecutive integers with one of which almost-prime. In [25], the second named author first exploited this problem and proved that there exists a strictly positive constant $C(\beta)$ such that

$$
\sum_{\substack{n \leqslant x, P^{-}(n)>x^{\beta} \\ P^{+}(n)<P^{+}(n+2)}} 1 \geqslant\{C(\beta)+o(1)\} \frac{x}{\log x}
$$

for $0<\beta<\frac{1}{3}$. Taking $\beta=\frac{1}{3}-\delta$ with $0<\delta \leqslant \frac{1}{12}$ in (1.8), we obtain the lower bound

$$
\sum_{\substack{P_{3} \leqslant x, P^{-}\left(P_{3}\right)>x^{1 / 3-\delta} \\ P^{+}\left(P_{3}\right)<P^{+}\left(P_{3}+2\right)}} 1 \geqslant\left\{C\left(\frac{1}{3}-\delta\right)+o(1)\right\} \frac{x}{\log x}
$$

for $0<\delta \leqslant \frac{1}{12}$. Here $P_{3}$ denotes an integer having at most three prime factors counted with multiplicity, i.e. $P_{3}$ is an almost-prime. The most interesting case in this problem is undoubtedly on primes, i.e. replacing $P_{3}$ in (1.9) by a prime number $p$, or equivalently, 
extend the value of $\beta$ in (1.8) to $0<\beta \leqslant \frac{1}{2}$, considering the well-known twin prime conjecture is equivalent to

$$
\left|\left\{p \leqslant x: P^{+}(p)<P^{+}(p+2)\right\}\right| \rightarrow \infty \text { for } x \rightarrow \infty .
$$

In addition, it should be noted that we can obtain a result similar to (1.8) directly for the small values of $\beta$ by a result of Fouvry [18, Théorème 2], a theorem of Bombieri-Vinogradov type on sifted integers (without small prime factor) with an exponent of distribution $>\frac{1}{2}$, or applying a weighted sieve combined with a theorem of Bombieri-Vinogradov type (see Fouvry [8, Théorème 2] and [7, Corollaire 3]).

In this paper, we treat the dual problem of (1.8), i.e. we replace the condition $P^{+}(n)<$ $P^{+}(n+2)$ by $P^{+}(n)>P^{+}(n+2)$ with $n$ free of small prime factor. In fact, this problem was posed by Fouvry in the thesis report that he wrote for the second named author. With the help of (1.5), and the lower bound of $\Phi(x, y, z)$ in Theorem 1, we prove that there exists a positive density of $n$ such that $P^{+}(n)>P^{+}(n+2), P^{-}(n)>x^{\alpha}$ with $0<\alpha<\frac{1}{4}$ :

Corollary 1. For $x \rightarrow \infty$ and $0<\alpha<\frac{1}{4}$, there exists a strictly positive constant $C_{1}(\alpha)$ such that

$$
\sum_{\substack{n \leqslant x, P^{-}(n)>x^{\alpha} \\ P^{+}(n)>P^{+}(n+2)}} 1 \geqslant\left\{C_{1}(\alpha)+o(1)\right\} \frac{x}{\log x} .
$$

It would be interesting to extend the domain of $\alpha$ to $0<\alpha<\frac{1}{2}$.

\section{LEMMAS}

The first lemma is the Rosser-Iwaniec sieve [16]. Here we only employ the upper-bound sieve.

Lemma 2.1. Let $D \geqslant 2$ and $L>1$. Let $\mathscr{P}$ denote a set of primes, let $z \geqslant 2$ and write $P(z):=\prod_{p \leqslant z, p \in \mathscr{P}} p$. There exists two sequences $\left\{\lambda_{d}^{ \pm}\right\}_{d=1}^{\infty}$ of real numbers, vanishing for $d>D$ or $\mu(d)=0$, satisfying $\lambda_{1}^{ \pm}=1,\left|\lambda_{d}^{ \pm}\right| \leqslant 1, \lambda^{-} * \mathbf{1} \leqslant \mu * \mathbf{1} \leqslant \lambda^{+} * \mathbf{1}$, and such that

$$
\begin{gathered}
\sum_{d \mid P(z)} \lambda_{d}^{+} \frac{w(d)}{d} \leqslant \prod_{\substack{p \leqslant z \\
p \in \mathscr{P}}}\left(1-\frac{w(p)}{p}\right)\left\{F(s)+O\left(\frac{e^{L^{1 / 2}-s}}{(\log D)^{1 / 3}}\right)\right\}, \\
\sum_{d \mid P(z)} \lambda_{d}^{-} \frac{w(d)}{d} \geqslant \prod_{\substack{p \leqslant z \\
p \in \mathscr{P}}}\left(1-\frac{w(p)}{p}\right)\left\{f(s)-O\left(\frac{e^{L^{1 / 2}-s}}{(\log D)^{1 / 3}}\right)\right\}
\end{gathered}
$$

uniformly for all multiplicative functions $w$ satisfying

(i) $0<w(p)<p \quad(p \in \mathscr{P})$,

(ii) $\prod_{\substack{u<p \leqslant v \\ p \in \mathscr{P}}}\left(1-\frac{w(p)}{p}\right)^{-1} \leqslant \frac{\log v}{\log u}\left(1+\frac{L}{\log u}\right) \quad(2 \leqslant u \leqslant v \leqslant z)$. 
where $s=\log D / \log z$ and $F(s), f(s)$ are defined by the continuous solutions to the system

$$
\begin{cases}s F(s)=2 \mathrm{e}^{\gamma} & 1 \leqslant s \leqslant 2 \\ s f(s)=0 & 0<s \leqslant 2 \\ (s F(s))^{\prime}=f(s-1) & s>2 \\ (s f(s))^{\prime}=F(s-1) & s>2 .\end{cases}
$$

Here $\gamma$ is the Euler constant.

Define

$$
S(x, y):=\left\{n \leqslant x: P^{+}(n) \leqslant y\right\}, \quad \Psi(x, y):=|S(x, y)|
$$

and

$$
\Psi(x, y ; a, q):=\sum_{\substack{n \in S(x, y) \\ n \equiv a(\bmod q)}} 1, \quad \Psi_{q}(x, y):=\sum_{\substack{n \in S(x, y) \\(n, q)=1}} 1 .
$$

The following two lemmas are on the distribution of friable numbers, obtained by Hildebrand [15, Theorem 1] and Fouvry-Tenenbaum [10, Théorème 1] respectively.

Lemma 2.2. For $\varepsilon>0$, we have

$$
\Psi(x, y)=x \rho(u)\left\{1+O_{\varepsilon}\left(\frac{\log (u+1)}{\log y}\right)\right\}
$$

uniformly for

$$
x \geqslant x_{0}(\varepsilon), \quad \exp \left\{\left(\log _{2} x\right)^{5 / 3+\varepsilon}\right\} \leqslant y \leqslant x,
$$

where $u=\log x / \log y$ and $\rho(u)$ is the Dickman-de Bruijn function.

Lemma 2.3. For $\varepsilon>0$, we have

$$
\Psi_{q}(x, y)=\frac{\varphi(q)}{q} \Psi(x, y)\left\{1+O\left(\frac{\log _{2}(q y) \log _{2} x}{\log y}\right)\right\}
$$

uniformly for

$$
x \geqslant x_{0}(\varepsilon), \quad \exp \left\{\left(\log _{2} x\right)^{5 / 3+\varepsilon}\right\} \leqslant y \leqslant x
$$

and

$$
\log _{2}(q+2) \leqslant\left(\frac{\log y}{\log (u+1)}\right)^{1-\varepsilon},
$$

where $u=\log x / \log y$ and $\varphi(n)$ is the Euler function.

The fourth lemma is the Bombieri-Vinogradov theorem for friable numbers, concerning the distribution of friable numbers in arithmetic progressions, averaged over a range of moduli. For $\Psi(x, y ; a, q)$ and $\Psi_{q}(x, y)$ defined by $(2.2)$, we have

Lemma 2.4. For any $A>0$, there exists a constant $B=B(A)>0$ such that

$$
\sum_{q \leqslant x^{1 / 2} /(\log x)^{B}} \max _{x^{\prime} \leqslant x} \max _{(a, q)=1}\left|\Psi\left(x^{\prime}, y ; a, q\right)-\frac{\Psi_{q}\left(x^{\prime}, y\right)}{\varphi(q)}\right| \ll_{A} \frac{x}{(\log x)^{A}}
$$

uniformly for $x \geqslant y \geqslant 2$. 
In [26], Wolke proved a theorem of Bombieri-Vinogradov for sifted numbers, and announced Lemma 2.4. In 1991, Fouvry and Tenenbaum [10] proved Lemma 2.4 with a different method. In addition, for Lemma 2.4, we can see the work of Fouvry and Tenenbaum (also the work of Drappeau [4]) with the level of the distribution of friable numbers $>1 / 2$ for some small $y$, or the work of Harper [14].

\section{Proof of Theorem 1}

For $\Phi(x, y, z)$ defined by (1.3) with $y=x^{1 / u}, z=x^{1 / v}$ and any $a \in \mathbb{Z}^{*}$ fixed, we apply Lemma 2.1 by taking

$$
\mathscr{P}=\{p: p \text { is prime, } p \nmid a\} \text {. }
$$

So we have

$$
\begin{aligned}
& \Phi(x, y, z)=\sum_{\substack{n \leqslant x+a,(n, a)=1 \\
P^{+}(n-a) \leqslant y \\
(n, P(z))=1}} 1+O(1) \\
& =\sum_{\substack{n \leqslant x+a,(n, a)=1 \\
P^{+}(n-a) \leqslant y}} \mu * \mathbf{1}((n, P(z)))+O(1) \\
& \geqslant \sum_{d \mid P(z)} \lambda_{d}^{-} \sum_{\substack{n \leqslant x+a,(n, a)=1 \\
P^{+}(n-a) \leqslant y \\
n \equiv 0(\bmod d)}} 1+O(1) \\
& =\sum_{d \mid P(z)} \lambda_{d}^{-} \sum_{\substack{n \leqslant x,(n, a)=1 \\
P^{+}(n) \leqslant y \\
n \equiv-a(\bmod d)}} 1+O(1) .
\end{aligned}
$$

Then we write this in the following form

$$
\Phi(x, y, z) \geqslant M(x, y, z)+R(x, y, z)+O(1)
$$

where

$$
M(x, y, z):=\sum_{d \leqslant D, d \mid P(z)} \frac{\lambda_{d}^{-}}{\varphi(d)} \sum_{\substack{n \leqslant x,(n, a)=1 \\ P^{+}(n) \leqslant y \\(n, d)=1}} 1
$$

and

$$
R(x, y, z):=\sum_{d \leqslant D, d \mid P(z)} \lambda_{d}^{-}\left(\sum_{\substack{n \leqslant x,(n, a)=1 \\ P^{+}(n) \leqslant y \\ n \equiv-a(\bmod d) \\ 5}} 1-\frac{1}{\varphi(d)} \sum_{\substack{n \leqslant x,(n, a)=1 \\ P^{+}(n) \leqslant y \\(n, d)=1}} 1\right)
$$


Taking $D=x^{1 / 2} /(\log x)^{B}$ for convenable $B>0$, we see that the error term $R(x, y, z)$ is admissible with the help of Lemma 2.4

$$
\begin{aligned}
R(x, y, z) & =\sum_{d \leqslant D, d \mid P(z)} \lambda_{d}^{-}\left(\sum_{\substack{n \leqslant x, P^{+}(n) \leqslant y \\
n \equiv-a(\bmod d)}} 1-\frac{1}{\varphi(d)} \sum_{\substack{n \leqslant x, P^{+}(n) \leqslant y \\
(n, d)=1}} 1\right) \sum_{\ell \mid(n, a)} \mu(\ell) \\
& =\sum_{d \leqslant D, d \mid P(z)} \lambda_{d}^{-} \sum_{\ell \mid a} \mu(\ell)\left(\sum_{\substack{m \leqslant x / \ell, P^{+}(m) \leqslant y \\
m \equiv-a \bar{\ell}(\bmod d)}} 1-\frac{1}{\varphi(d)} \sum_{\substack{m \leqslant x / \ell, P^{+}(m) \leqslant y \\
(m, d)=1}} 1\right) \\
& \ll \sum_{d \leqslant D} \max _{x^{\prime} \leqslant x} \max _{\left(a^{\prime}, d\right)=1}\left|\Psi\left(x^{\prime}, y ; a^{\prime}, d\right)-\frac{\Psi_{d}\left(x^{\prime}, y\right)}{\varphi(d)}\right| \\
& \ll \frac{x}{(\log x)^{A}}
\end{aligned}
$$

for any $A>0$ and any fixed integer $a \neq 0$.

Now it remains to estimate $M(x, y, z)$. We first change the order of summation and write

$$
M(x, y, z)=\sum_{\substack{n \leqslant x,(n, a)=1 \\ P^{+}(n) \leqslant y}} \sum_{\substack{d \leqslant D \\ d \mid P^{\prime}(z)}} \frac{\lambda_{d}^{-}}{\varphi(d)}
$$

where $P^{\prime}(z)=\prod_{p<z, p \in \mathscr{P}^{\prime}} p$ with $\mathscr{P}^{\prime}:=\{p: p \nmid a n\}$. Note that by (3.1) we may deduce that $n$ and $a$ have opposite parity which implies $2 \mid a n$. We apply Lemma 2.1 with $w(p)=p / \varphi(p)$ and some large constant $L$ getting

$$
\begin{aligned}
M(x, y, z) & \geqslant\left\{f\left(\frac{v}{2}\right)-o(1)\right\} \sum_{\substack{n \leqslant x,(n, a)=1 \\
P^{+}(n) \leqslant y}} \prod_{\substack{2<p \leqslant z \\
p \nmid a n}}\left(1-\frac{1}{p-1}\right) \\
& =\left\{f\left(\frac{v}{2}\right)-o(1)\right\} \prod_{\substack{2<p \leqslant z \\
p \nmid a}}\left(\frac{p-2}{p-1}\right) \sum_{\substack{n \leqslant x,(n, a)=1 \\
P^{+}(n) \leqslant y}} H(n)
\end{aligned}
$$

where $H(n)$ is a multiplicative function defined by

$$
H(n)=\prod_{2<p \leqslant z, p \mid n}\left(\frac{p-1}{p-2}\right)
$$

By the following formula due to Liu-Wu-Xi (see [17, (3.5)])

$$
\sum_{\substack{n \leqslant x,(n, a)=1 \\ P^{+}(n) \leqslant y}} H(n)=\frac{\varphi(a)}{a} \prod_{\substack{2<p \leqslant z \\ p \nmid a}}\left(1+\frac{1}{p(p-2)}\right) \Psi(x, y)\left\{1+O\left(\frac{(\log \log x)^{2}}{\log y}\right)\right\}
$$


we arrive at by Mertens' formula and Lemma 2.2

$$
\begin{aligned}
M(x, y, z) & \geqslant\left\{f\left(\frac{v}{2}\right)-o(1)\right\} \frac{\varphi(a)}{a} \prod_{\substack{2<p \leqslant z \\
p \nmid a}}\left(1-\frac{1}{p}\right) \Psi(x, y) \\
& \geqslant\left\{f\left(\frac{v}{2}\right)-o(1)\right\} \frac{\mathrm{e}^{-\gamma}}{\log z} \Psi(x, y) \\
& =\left\{f\left(\frac{v}{2}\right)-o(1)\right\} \frac{v \mathrm{e}^{-\gamma}}{\log x} \rho(u) x .
\end{aligned}
$$

We remark that if $a$ is odd, we may obtain a better lower bound with an extra constant factor 2 .

Finally, combining (3.2), (3.3) and (3.4), we obtain

$$
\Phi(x, y, z) \geqslant v \rho(u) f\left(\frac{v}{2}\right) \frac{\mathrm{e}^{-\gamma} x}{\log x}\{1-o(1)\}
$$

for $y=x^{1 / u}, z=x^{1 / v}$ with $v>4,1 \leqslant u \ll 1$. This completes the proof of Theorem 1 .

\section{Proof of Corollary 1}

For $0<\alpha<\frac{1}{4}$, we have

$$
\sum_{\substack{n \leqslant x, P^{-}(n)>x^{\alpha} \\ P^{+}(n)>P^{+}(n+2)}} 1 \geqslant \sum_{\substack{n \leqslant x, P^{-}(n)>x^{\alpha} \\ P^{+}(n+2)<x^{\alpha}}} 1 .
$$

By taking $z=x^{\alpha}$ and $a=-2$ in (1.5) of Theorem 1, we obtain Corollary 1.

Acknowledgements The authors first are very grateful to Professor Valentin Blomer for pointing out a mistake in the original version of the paper [18]. The authors would like to express the most sincere gratitude to Professor Étienne Fouvry for posing the question in Corollary 1, which is the original motivation of this paper. The authors would also like to thank Professors Cecile Dartyge and Jie Wu for their valuable advice and constant encouragement. The second author is supported by the China Postdoctoral Science Foundation (No. 2017M621829), the Jiangsu Province Postdoctoral Science Foundation (No. 1701142C) and the Yangzhou University Overseas Study Program. The first and third authors are supported by China Scholarship Council, and the third author is partly supported by the NSFC(61402335), SRPF-SXPED(17JK0266), NSF-WNU(17ZRRC01).

\section{REFERENCES}

[1] R. C. Baker and G. Harman. The Brun-Titchmarsh theorem on average. In Analytic number theory, Vol. 1 (Allerton Park, IL, 1995), volume 138 of Progr. Math., pages 39-103. Birkhäuser Boston, Boston, MA, 1996.

[2] W. D. Banks and I. E. Shparlinski. On values taken by the largest prime factor of shifted primes. Journal of the Australian Mathematical Society, 82(1):133-147, 2007.

[3] F.-R. Chen and Y.-G. Chen. On the largest prime factor of shifted primes. Acta Math. Sinica, English Series, 3:377-382, 2017. 
[4] S. Drappeau. Théorèmes de type Fouvry-Iwaniec pour les entiers friables. Compos. Math., 151(05):828$862,2015$.

[5] P. Erdôs. On the normal number of prime factors of $p-1$ and some related problems concerning Euler's $\phi$-function. Quart. J. Math., 6:205-213, 1935.

[6] B. Feng and J. Wu. On the density of shifted primes with large prime factors. Sci. China Math., 61(1):83-94, 2018.

[7] E. Fouvry. Répartition des suites dans les progressions arithmétiques. Acta Arith., 4(41):359-382, 1982.

[8] E. Fouvry. Autour du théorème de Bombieri-Vinogradov. Acta Math., 152(1):219-244, 1984.

[9] E. Fouvry. Théorème de Brun-Titchmarsh; application au théorème de Fermat. Invent. Math., 79(2):383-407, 1985.

[10] E. Fouvry and G. Tenenbaum. Entiers sans grand facteur premier en progressions arithmétiques. Proc. Lond. Math. Soc., 3(63):449-494, 1991.

[11] J. B. Friedlander. Integers free from large and small primes. Proc. Lond. Math. Soc., 3(3):565-576, 1976.

[12] M. Goldfeld. On the number of primes $p$ for which $p+a$ has a large prime factor. Mathematika, 16:23-27, 1969.

[13] A. Granville. Smooth numbers: computational number theory and beyond. Algorithmic number theory: lattices, number fields, curves and cryptography, 44:267-323, 2008.

[14] A. J. Harper. Bombieri-Vinogradov and Barban-Davenport-Halberstam type theorems for smooth numbers. arXiv:1208.5992, preprint(2012).

[15] A. Hildebrand. On the number of positive integers $\leqslant x$ and free of prime factors $>y$. J. Number Theory, 22:289-307, 1986.

[16] H. Iwaniec. A new form of the error term in the linear sieve. Acta Arith., 37:307-320, 1980.

[17] J. Liu, Wu. J., and Xi P. Primes in arithmetic progressions with friable indices. Sci. China Math., 63:23-38, 2020.

[18] Xiaodong Lü, Zhiwei Wang, and Bin Chen. On the smooth values of shifted almost-primes. Int. J. Number Theory, 15(1):1-9, 2019.

[19] F. Luca, R. Menares, and A. Pizarro-Madariaga. On shifted primes with large prime factors and their products. Bull. Belg. Math. Soc. Simon Stevin, 22:39-47, 2015.

[20] C. Pomerance. Popular values of Euler's function. Mathematika, 27(01):84-89, 1980.

[21] E. Saias. Entiers sans grand ni petit facteur premier I. Acta Arith., 61(3):347-374, 1992.

[22] E. Saias. Entiers sans grand ni petit facteur premier II. Acta Arith., 63(4):287-312, 1993.

[23] E. Saias. Entiers sans grand ni petit facteur premier III. Acta Arith, 71(4):351-379, 1995.

[24] G. Tenenbaum. Introduction à la théorie analytique et probabiliste des nombres, 3ième édition. Collection Échelles, Édition Belin, 2008.

[25] Z. W. Wang. Autour des plus grands facteurs premiers d'entiers consécutifs voisins d'un entier criblé. Quart. J. Math., to appear.

[26] D. Wolke. Über die mittlere verteilung der werte zahlentheoretischer funktionen auf restklassen. II. Math. Ann., 204(2):145-153, 1973.

Xiaodong Lü, School of Mathematical Science, Yangzhou University, 225002, Yangzhou, CHINA,

Email address: xidolv@gmail.com

Zhiwei Wang, Institut Élie Cartan de Lorraine, Université de Lorraine, UMR 7502, 54506 VANDEUVRE-LÈS-NANCY, FranCE

Email address: wangzhiwei.sdu@gmail.com

Bin Chen, School of mathematics, Shandong University, Shanda Road, 250100, Jinan, China, School of mathematics and Physics, Weinan Normal University, Chaoyang Road, 714099, Weinan, China,

Email address: 13tjccbb@tongji.edu.cn 